\title{
Bisphosphonates: from bone anti-resorptive to anti-cancer drugs.
}

\author{
Mohammed Helmy Faris Shalayel ${ }^{1,2 *}$, Saeed Ali Alsareii ${ }^{3}$, Abdulhadi Mohamed Elbashir ${ }^{3}$, Mohammed Ayed \\ Huneif $^{4}$ \\ ${ }^{1}$ Professor of Biochemistry and Dermatovenereologist, Nile College, Sudan \\ ${ }^{2}$ College of Medicine, Najran University, Saudi Arabia \\ ${ }^{3}$ Assistant Professor of Surgery, College of Medicine, Najran University, Saudi Arabia \\ ${ }^{4}$ Assistant Professor of Pediatrics, College of Medicine, Najran University, Saudi Arabia
}

\begin{abstract}
Bisphosphonates, analogues of pyrophosphates, are utilized as efficient drugs against bone resorption in bone disorders like, osteoporosis, Paget's disease, multiple myeloma, cancerinduced hypercalcemia, and bony metastases. They suppress osteoclastic bone resorption by incorporating with hydroxyapatite binding sites on bony surfaces that undergo active resorption with due interference with various biochemical processes in bone-resorbing osteoclasts, impairing the ability of the osteoclasts and enhancing osteoclast apoptosis. The clinical utilization of bisphosphonates has dramatically expanded during the past 3 decades or more especially for osteoporosis to diminish the frequency of skeletal-related events in patients with breast cancer and myeloma. Some preclinical studies had reported that bisphosphonates exhibit direct and indirect anticancer activities in patients with early breast cancer or symptomatic multiple myeloma exhibiting disease-free survival and overall survival benefits. Moreover, some epidemiological and clinically applied studies concluded that current use of bisphosphonates in healthy postmenopausal women to manage osteoporosis was correlated with a $30 \%$ reduction in the risk of breast and colon cancers and ladies who use bisphosphonates had about one half the risk of getting endometrial cancer compared with those who did not use them. Recently, it was reported that addition of bisphosphonates to epidermal growth factor receptor-tyrosine kinase inhibitors (EGFR-TKIs) could enhance the antitumor effect of EGFR-TKIs in patients with EGFR-mutant non-small-cell lung cancer and bone metastasis.
\end{abstract}

Keywords: Bisphosphonates, Multiple myeloma, Osteoclast, Pamidronate, Zoledronate.

Accepted on April 22, 2017

Bisphosphonates are structurally stable derivatives of inorganic pyrophosphate (PPi) [1] but are chemically reluctant to enzymatic breakdown [2]. It was suggested that inorganic pyrophosphate might be the bodies own natural "water softener" that normally prohibits soft tissues calcification and organizes mineralization of bones. Hence, it became clear that calcification disorders might be associated with defects in PPi metabolism [3]. Bisphosphonates are analogues to a metabolic byproduct compound with 2 phosphate groups connected together by esterification, the so-called pyrophosphate. They have sturdy inhibitory influence on bone resorption. Consequently, they are regarded as efficient drugs against bone resorption in bone disorders like, osteoporosis, Paget's disease, multiple myeloma, cancer-induced hypercalcemia, and bony metastases [4].

Resembling their natural analogue PPi, bisphosphonates have a very high affinity for bone mineral as they bind to hydroxyapatite crystals. Therefore, availability of hydroxyapatite binding sites is crucial in bisphosphonate skeletal retention. Bisphosphonates are discriminatorily commingle places of active bone remodeling, as commonly happens in conditions characterized by accelerated skeletal turnover. That are not retained in the skeleton, are rapidly washed out from the circulation by renal clearance. Additionally, they effectively suppress bone resorption by inhibiting hydroxyapatite breakdown [5].

The bisphosphonates suppress osteoclastic bone resorption through a mechanism that differs from that of other antiresorptive compounds [6,7]. They incorporate with hydroxyapatite binding sites on bony surfaces that succumb active resorption impairing the ability of the osteoclasts to form the ruffled border and to produce the protons necessary for sustained bone resorption [7-9]. Bisphosphonates also reduce osteoclast activity by enhancing osteoclast apoptosis and diminishing osteoclast progenitor development and recruitment [10]. In addition, bisphosphonates interfere 
with various biochemical processes in bone-resorbing osteoclasts after selective adsorption to mineral surfaces and subsequent internalization by osteoclasts [2]. Moreover, they can improve the biomechanical properties of bone in both normal animals and experimental models of osteoporosis if not given in excess [11].

The P-C-P part of the bisphosphonates is responsible for the robust affinity for binding to hydroxyapatite and permits for a number of diversities in structure on the basis of substitution in the R1 and R2 positions on the carbon atom [12]. Some experimental studies suggested that bisphosphonates may conserve osteocytes and osteoblasts against glucocorticoidsinduced apoptosis [13]. Recent evidence proposed that the inhibition of osteocyte apoptosis by bisphosphonates is interposed through the conquering of connexion 43 hemichannels and brisking of extracellular signal-regulated kinases [14,15].

Nitrogenous (N-containing) bisphosphonates include Alendronate, Ibandronate, Neridronate, Olpadronate, Pamidronate, Risedronate and Zoledronate while Nonnitrogenous (Non-N-containing) bisphosphonates include Clodronate, Etidronate and Tiludronate [16,17]. The nonnitrogenous bisphosphonates are metabolized within the cell to certain metabolites that substitute the terminal pyrophosphate moiety of adenosine triphosphate (ATP), producing a non-functional molecule that contends with ATP in the cellular energy metabolism. Duly, the osteoclasts commence apoptosis, leading to an overall bone resorption decrease [18]. Whereas, nitrogen containing bisphosphonates are suggested to restrain osteoclast activity by blocking the enzyme farnesyl diphosphate synthase in the HMG-CoA reductase pathway [19].

The clinical employment of bisphosphonates has dramatically grown during the past 3 decades especially for osteoporosis. The Food and Drug Administration's approval of pamidronate in 1995 for the remediation of normocalcemic patients with myeloma and in 1996 for women who suffered from osteolytic lesions due to metastatic breast cancer was followed by widespread use of pamidronate in patients with cancers that encompass bone. Hence, it was suggested that $\mathrm{N}$-containing bisphosphonate, pamidronate, diminishes the frequency of skeletal-related events in patients with breast cancer or myeloma [20].

Randomized clinical trials evaluated the role of bisphosphonate adjuvant therapy in cancer are still in progress. In vitro and in vivo initial studies proposed that bisphosphonates play direct and indirect roles as antitumor drugs. Interestingly, some clinical data confirmed these conclusions in cancer patients with bone metastasis especially breast cancer [21-23].

Although, results from clinical trials assaying the anticancer efficiency of bisphosphonates have been conflicting, they generally uphold a small absolute improvement in overall survival, with the largest treatment impact on diminishing the bone metastasis recurrence [24]. Nevertheless, Vale et al. [25] showed that in prostate cancer with locally advanced disease, there is no evidence of survival benefits from bisphosphonates addition. However, treatment with bisphosphonates was previously indicated in men with prostate cancer who were castrated or received gonadotropin releasing hormone agonists and was at risk of osteoporosis [26]. Contrastingly, some preclinical studies had showed that bisphosphonates exhibit direct and indirect anticancer activities in patients with early breast cancer or symptomatic multiple myeloma exhibiting disease-free survival and overall survival benefits [27].

In a randomized study, prostate cancer patients were treated with these gonadotropin releasing hormone agonists, alone or with pamidronate. The pamidronate-treated patients did not lose bone mineral density, whereas patients treated with the gonadotropin-releasing hormone agonist alone experienced significant bone loss [28]. Solid tumors (lung cancer, breast cancer, prostate cancer) and multiple myeloma are liable to develop bone metastasis. In the bone marrow, tumor cells do not devastate bone rather than altering the functions of osteoclasts and osteoblasts, and hijacking advent signals from the bone matrix. Thus, tumor cells block bone formation and consolidate bone resorption leading to skeletal destruction and subsequent occurrence of skeletal events [29]. These skeletal events can be lethal and have great impact on patients by causing hypercalcemia, pathological fractures, spinal cord compression and mobility loss [30].

Owing to their sturdy anti-resorptive efficacy, bisphosphonates (especially $\mathrm{N}$-containing bisphosphonates) are therefore used to treat malignant bone diseases and delay skeletal events associated with bone metastasis [31]. Thence, there may be a growing justification for the use of bisphosphonates than has previously been considered.

Concordantly, some epidemiological studies concluded that current use of bisphosphonates in healthy postmenopausal ladies to manage osteoporosis was correlated with a $30 \%$ reduction in the risk of breast and colon cancers. These conclusions highlight the significance of identifying mechanisms that are responsible for the anticancer efficacy of some bisphosphonates like zoledronate and clodronate against breast cancer under estrogen indigence. Understanding these distinct mechanisms beyond the fact that zoledronate improved cancer-related outcomes in patients with newly diagnosed multiple myeloma as well as in patients with breast cancer, will assist to better recognize patients who could get benefits from treatment with bisphosphonate [26].

Some investigators reported a significant reduction (32 vs. $63 ; \mathrm{P}<0.005)$ in the total number of bone metastases in stage IV breast cancer patients without skeletal metastases after placebo administration of $1,600 \mathrm{mg}$ clodronate for 3 years [32]. Due to the poor oral bioavailability of pamidronate, a similar trial of its oral administration failed to emphasize these findings [33].

There is growing preclinical evidence suggesting that adjuvant protocols utilizing $\mathrm{N}$-containing bisphosphonates in combination therapy results in increased antitumor effects against breast tumors and improved survival [34]. Moreover, a recent proposal suggested that ladies who use bisphosphonates had about one half the risk of getting endometrial cancer compared with ladies who did not use 
these medications. Alford et al. [35] assessed whether bisphosphonates might help prevent endometrial cancer. The researchers evaluated information from the National Cancer Institute's PLCO (Prostate, Lung, Colorectal and Ovarian) Screening Trial, which included questionnaires about bone medication use. Data were analyzed only for bisphosphonates that contain nitrogen, which are known to have strong anticancer activity. The study included 29,254 women. After factors such as age, race, history of hormone therapy use, smoking status and body mass index had been taken into account, bisphosphonate users were half as likely to develop endometrial cancer.

Zhang et al. [36] reported that epidermal growth factor receptor (EGFR) mutation was the significant independent prognostic factor for overall survival and the addition of bisphosphonates to epidermal growth factor receptor -tyrosine kinase inhibitors (EGFR-TKIs) could enhance the antitumor effect of EGFR-TKIs in patients with EGFRmutant non-small-cell lung cancer and bone metastasis.

\section{Conclusion}

Bisphosphonates are anti-resorptive drugs that widely utilized in osteoporosis, Paget's disease, multiple myeloma, cancerinduced hypercalcemia, and bony metastases to suppress osteoclastic bone resorption. Due to their powerful antiresorptive efficacy, bisphosphonates (especially N-containing bisphosphonates) are therefore used to treat malignant bone diseases and delay skeletal events associated with bone metastasis. There is a strong evidence that bisphosphonates exhibit direct and indirect anticancer activities in patients with prostate cancer, early breast cancer or symptomatic multiple myeloma exhibiting some overall survival benefits. Concordantly, adjuvant protocols utilizing N-containing bisphosphonates in combination therapy resulted in increased antitumor effects against breast tumors and improved survival.

\section{References}

1. Russell RG. Bisphosphonates: From bench to bedside. Ann N Y Acad Sci. 2006;1068:367-401.

2. Russell RG. Bisphosphonates: Mode of action and pharmacology. Pediatrics. 2007;119(Suppl 2):S150-62.

3. Russell RG. Excretion of inorganic pyrophosphate in hypophosphatasia. Lancet. 1965;10:461-4.

4. Martin TJ, Grill V. Bisphosphonates - Mechanisms of action. Exp Clin Pharmacol. 2000;23(6):130-2.

5. Drake MT, Clarke BL, Khosla S. Bisphosphonates: Mechanism of action and role in clinical practice. Mayo Clin Proc. 2008;83(9):1032-45.

6. Fleisch H. Bisphosphonates: Mechanisms of action. Endocr Rev. 1998;19(1):80-100.

7. Colucci S, Minielli V, Zambonin G, et al. Alendronate reduces adhesion of human osteoclast-like cells to bone and bone protein-coated surfaces. Calcif Tissue Int. 1998;63(3):230-5.

8. Hughes DE, Wright KR, Uy HL, et al. Bisphosphonates promote apoptosis in murine osteoclasts in vitro and in vivo. J Bone Miner Res. 1995;10(10):1478-87.

9. Sato M, Grasser W, Endo N, et al. Bisphosphonate action. Alendronate localization in rat bone and effects on osteoclast ultrastructure. J Clin Invest. 1991;88(6):2095105.

10. https://www.uptodate.com/contents/pharmacology-ofbisphosphonates

11. Ferretti JL. Effects of biosphosphonates on bone biomechanics. In: Bijvoet O, Fleisch HA, Canfield RE, Russell RG (Eds.), Bisphosphonates on Bone. Elsevier Science, Amsterdam, Holland. 1995;211-29.

12. Ebrahimpour A, Francis MD. Bisphosphonate therapy in acute and chronic bone loss: Physical chemical considerations in bisphosphonate-related therapies. In: Bijvoet O, Fleisch HA, Canfield RE, Russell RG (Eds.), Bisphosphonates on Bones. Elsevier Science, Amsterdam, Holland. 1995;125-36.

13. Plotkin LI, Weinstein RS, Parfitt AM, et al. Prevention of osteocyte and osteoblast apoptosis by bisphosphonates and calcitonin. J Clin Invest. 1999;104:1363-74.

14. Plotkin LI, Aguirre JI, Kousteni S, et al. Bisphosphonates and estrogens inhibit osteocyte apoptosis via distinct molecular mechanisms downstream of extracellular signal-regulated kinase activation. J Biol Chem. 2005;280:7317-25.

15. Lezcano V, Bellido T, Plotkin LI, et al. Role of connexin 43 in the mechanism of action of alendronate: Dissociation of anti-apoptotic and proliferative signaling pathways. Arch Biochem Biophys. 2012;518(2):95-102.

16. Frith J, Mönkkönen J, Blackburn G, et al. Clodronate and liposome-encapsulated clodronate are metabolized to a toxic ATP analog, adenosine 5'-(beta, gammadichloromethylene) triphosphate, by mammalian cells in vitro. J Bone Miner Res. 1997;12(9):1358-67.

17. van Beek E, Cohen L, Leroy I, et al. Differentiating the mechanisms of anti-resorptive action of nitrogen containing bisphosphonates. Bone 2003;33(5):805-11.

18. https://en.wikipedia.org/wiki/Bisphosphonate

19. http://www.uptodate.com/contents/the-use-ofbisphosphonates-in-patients-with-multiple-myeloma

20. Mundy GR, Yoneda T. Bisphosphonates as anticancer drugs. N Engl J Med. 1998;339:398-400.

21. Santini D, Fratto ME, Galluzzo S, et al. Are bisphosphonates the suitable anticancer drugs for the elderly? Crit Rev Oncol Hematol. 2009;69(1):83-94.

22. Coleman R, De BR, Eidtmann H, et al. Zoledronic acid (zoledronate) for postmenopausal women with early breast cancer receiving adjuvant letrozole (ZO-FAST study): Final 60 month results. Ann Oncol. 2013;24(2):398-405.

23. Brufsky AM, Harker WG, Beck JT, et al. Final 5-year results of Z-FAST trial: Adjuvant zoledronic acid maintains bone 
mass in postmenopausal breast cancer patients receiving letrozole. Cancer. 2012;118(5):1192-201.

24. Yardley DA. Pharmacologic management of bone-related complications and bone metastases in postmenopausal women with hormone receptor-positive breast cancer. Breast Cancer (Dove Med Press). 2016;8:73-82.

25. Vale CL, Burdett S, Rydzewska LH, et al. Addition of docetaxel or bisphosphonates to standard of care in men with localised or metastatic, hormone-sensitive prostate cancer: A systematic review and meta-analyses of aggregate data. Lancet Oncol. 2016;17:243-56.

26. Clézardin P. Mechanisms of action of bisphosphonates in oncology: A scientific concept evolving from antiresorptive to anticancer activities. Bonekey Reports. 2013;2:267.

27. Mittan D, Lee S, Miller E, et al: Bone loss following hypogonadism in men with prostate cancer treated with GnRH analogs. J Clin Endocrinol Metab. 2002;87:365661.

28. Smith MR, McGovern FJ, Zietman AL, et al. Pamidronate to prevent bone loss during androgen deprivation therapy for prostate cancer. N Engl J Med. 2001;345:948-55.

29. Weilbaecher KN, Guise TA, McCauley LK. Cancer to bone: a fatal attraction. Nat Rev Cancer. 2012;11:411-24.
30. Clézardin P. Bisphosphonates' antitumor activity: An unravelled side of a multifaceted drug class. Bone. 2011;48:71-9.

31. Coleman R, Gnant M, Morgan G, et al. Effects of bonetargeted agents on cancer progression and mortality. J Natl Cancer Inst. 2012;104:1059-67.

32. Kanis JA, Powles T, Paterson AH, et al: Clodronate decreases the frequency of skeletal metastases in women with breast cancer. Bone. 1996;19:663-7.

33. Ford JM, van Oosterom, Brincker $\mathrm{H}$, et al. Oral pamidronate: Negative results from three double-blind, placebo controlled trials in hypercalcemia, myeloma and the prevention of bone metastases (abstract). Bone. 1998;22(Suppl 3):58S.

34. Michailidou M, Holen I. Combinations of bisphosphonates and classical anticancer drugs: A preclinical perspective. Prevention of Bone Metastases. 2012;192:145-68.

35. Alford SH, Rattan R, Buekers T, et al. Protective effect of bisphosphonates on endometrial cancer incidence in data from the prostate, lung, colorectal and ovarian (PLCO) cancer screening trial. Cancer. 2015;121(3):441-7.

36. Zhang G, Cheng R, Zhang Z, et al. Bisphosphonates enhance antitumor effect of EGFR-TKIs in patients with advanced EGFR mutant NSCLC and bone metastases. Sci Rep. 2017;7:42979.

\section{*Correspondence to:}

Mohammed Helmy Faris Shalayel

College of Medicine

Najran University

Saudi Arabia

Tel: +966-541476862

E-mail: drmhfs@hotmail.com 